\title{
Genome Analysis
}

\section{PyRanges: efficient comparison of genomic}

\section{intervals in Python}

\author{
Endre Bakken Stovner ${ }^{1,2,3,4 *}$ and Pål Sætrom ${ }^{1,2,3,4}$ \\ 1Department of Computer Science, Norwegian University of Science and Technology, Trondheim, 7013, \\ Norway, \\ 2Department of Clinical and Molecular Medicine, Norwegian University of Science and Technology, \\ Trondheim, 7013, Norway, \\ 3Bioinformatics Core Facility, Norwegian University of Science and Technology, Trondheim, 7013, Norway \\ ${ }^{4}$ K.G. Jebsen Center for Genetic Epidemiology, Department of Public Health and Nursing, Norwegian \\ University of Science and Technology, Trondheim, Norway \\ *To whom correspondence should be addressed. \\ Associate Editor: $X X X X X X X$ \\ Received on $X X X X X$; revised on $X X X X X$; accepted on $X X X X X$
}

\begin{abstract}
Summary: Complex genomic analyses often use sequences of simple set operations like intersection, overlap, and nearest on genomic intervals. These operations, coupled with some custom programming, allow a wide range of analyses to be performed. To this end, we have written PyRanges, a data structure for representing and manipulating genomic intervals and their associated data in Python. Run single-threaded on binary set operations, PyRanges is in median 2.3-9.6 times faster than the popular R GenomicRanges library and is equally memory efficient; run multi-threaded on 8 cores, our library is up to 123 times faster. PyRanges is therefore ideally suited both for individual analyses and as a foundation for future genomic libraries in Python.

Availability: PyRanges is available open-source under the MIT license at https://github.com/biocoreNTNU/pyranges and documentation exists at https://biocore-NTNU.github.io/pyranges/

Contact: endrebak85@gmail.com

Supplementary information: Supplementary data are available.
\end{abstract}


bioRxiv preprint doi: https://doi.org/10.1101/609396; this version posted April 16, 2019. The copyright holder for this preprint (which was not certified by peer review) is the author/funder, who has granted bioRxiv a license to display the preprint in perpetuity. It is made available under aCC-BY-ND 4.0 International license.

\section{E.B. Stovner et al.}

\section{Introduction}

Comparing sets of intervals is a fundamental task in genomics, and a few basic operations allow for answering many scientific questions. For example, to find genes potentially targeted by a transcription factor, one can intersect the sets of intervals representing gene positions and representing transcription factor binding sites to identify those that overlap.

Several toolboxes of genomic operations exist, such as bedtools (Quinlan et al., 2010) and bedops (Neph et al., 2012) for the command line and GenomicRanges (Lawrence et al., 2004) for the R programming environment.

GenomicRanges is a data structure for representing and operating on genomic intervals and their metadata, which are stored as a 2D-table in memory. By providing methods for access and for set operations on genomic intervals, programmers can use the $\mathrm{R}$ programming language to manipulate and analyse the contents of GenomicRanges. Consequently, GenomicRanges is a powerful tool for writing complex and custom genome analyses. Indeed, in R, GenomicRanges is a foundational library, and a cornerstone of genomics packages in the $\mathrm{R}$ Bioconductor project (Gentleman, 2004).

Python is currently ranked as the most popular programming language in the world (according to IEEE Spectrum's compound metric; S.Cass et. al, 2018) and is much used in data science and bioinformatics, yet it lacks a GenomicRanges implementation. The PyRanges library remedies this by providing a version which is multithreaded, fast, and memoryefficient.

\section{Library}

\section{Implementation}

The PyRanges data structure is logically represented as a 2D-table. Each row represents an interval, and the columns each describe either a part of the location (chromosome, start position, end position, and optionally, strand) or metadata (name, score, exon number or any arbitrary value desired by the user). The underlying implementation uses a dictionary that maps chromosome and strand pairs to their respective 2D-tables; however, this division is largely invisible to the user. The data in the 2Dtables are stored in Pandas DataFrames, thus allowing the vast Python science stack to be used seamlessly with PyRanges. Furthermore, DataFrames allow for storing the data contiguously in native data types, such as integers, floats, or categoricals, to ensure memory-efficiency. To make PyRanges fast, its operations are written in Cython or C. Moreover, by keeping the data belonging to each chromosome in separate DataFrames, these logically distinct data can easily be independently processed. For single threaded processing, this implementation detail has limited effect, but for multi threaded processing, we avoid the substantial time costs of splitting and merging the data for each operation. PyRanges provides parallel processing through the Ray framework (P.Moritz et al., 2017), resulting in a speedup provided the data are sufficiently big (see timings).

\section{Functionality}

PyRanges main functionality includes functions for reading genomic intervals from files, and unary and binary functions for manipulating one and two sets of genomic intervals. File reading functions support common formats such as bed, GTF/GFF, and bam. Unary functions manipulate single PyRanges by subsetting, clustering, or computing coverage; that is, the number of intervals overlapping each genomic position. Binary functions include operations such as intersection, nearest, and subtract that create a new set of genomic intervals by comparing two sets of intervals. See the Supplementary text for a full list of PyRanges' operations.

PyRanges also uses and provides two stand-alone libraries useful beyond bioinformatics. One library (pyrle) implements run-length encoding arithmetic, which is useful to compactly represent and efficiently do arithmetic on the coverage (or any other nucleotide-associated score) of sets of regions. The other library (NCLS) implements the Nested
Containment List, which is an immutable interval-tree with better memory-efficiency and speed than a regular interval-tree both for tree construction and interval queries (see Supplementary Timings)

\section{Performance}

The PyRanges library has been extensively benchmarked for both speed and memory use (Fig 1; Supplementary Timings). We used two types of data for testing: 1) libraries of reads only, i.e. they included no metadata and were hence more lightweight and 2) GTF annotations. We used unsorted test files generated by bedtools random for hg 38 to simulate the read files. To create a large GTF we used sampling with replacement on the Gencode hg38 GTF.

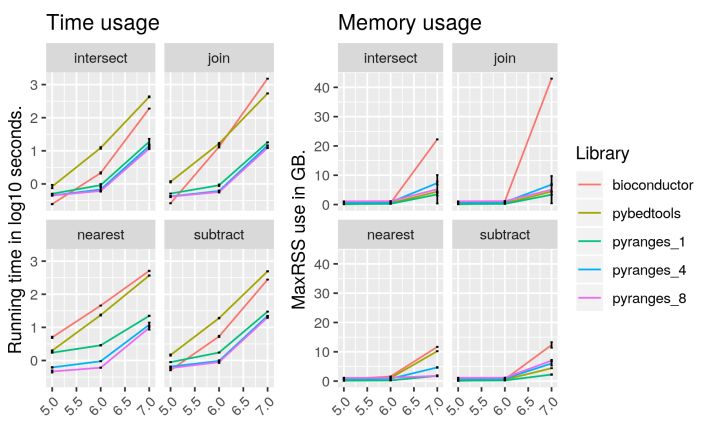

Log10 number of intervals.

Fig. 1 (Left) Running time and (Right) memory usage as a function of the number of intervals for four common binary functions on genomic intervals; see Supplementary Timings for complete benchmark results.

For binary operations, PyRanges in single-threaded mode was 6.5 - 31 (median 14) and 9.8 - 36 (median 24) times faster than pybedtools on $1 \mathrm{e} 6$ and $1 \mathrm{e} 7$ intervals, respectively. Compared to GenomcRanges, PyRanges was $1.3-16$ (median 2.3) and $1.9-84$ (median 9.6) times faster. Run multi-threaded on 8 cores, the speed-ups for the same operations on $1 \mathrm{e} 7$ intervals were $13-63$ and $1.8-123$ times compared to pybedtools and GenomicRanges, respectively. For all operations, PyRanges run single-threaded on $1 \mathrm{e} 7$ intervals had a median speed-up of 26 and 4.0 times and used a median 5.7 and 5.1 times less memory compared to pybedtools and GenomicRanges, respectively.

\section{Conclusion}

PyRanges is an efficient and feature rich library for genomics in the extremely popular Python programming language, and the only one of its kind. We therefore expect it to be a boon to current and future bioinformaticians and researchers working in Python.

Funding

This work was supported by the Research Council of Norway [grant number230338]; and Stiftelsen K.G. Jebsen.

Conflict of Interest: none declared.

\section{References}

R. C. Gentleman, V. J. Carey, D. M. Bates, B. Bolstad, M. Dettling, S. Dudoit, B. Ellis, L. Gautier, Y. Ge, J. Gentry, K. Hornik, T. Hothorn, W. Huber, S. Iacus, R. Irizarry, F. Leisch, C. Li, M. Maechler, A. J. Rossini, G. Sawitzki, C. Smith, G. Smyth, L. Tierney, J. Y. Yang, and J. Zhang. Bioconductor: open software development for computational biology and bioinformatics. Genome Biology, 5(10):R80, Sep 2004.

M. Lawrence, W. Huber, H. Pagès, P. Aboyoun, M. Carlson, R. Gentleman,

M. T. Morgan, and V. J. Carey. Software for computing and annotating genomic ranges. PLOS Computational Biology, 9(8):1-10, 082013.

S. Neph, M. S. Kuehn, A. P. Reynolds, E. Haugen, R. E. Thurman, A. K. Johnson, E. Rynes, M. T. Maurano, J. Vierstra, S. Thomas, R. Sandstrom, R. Humbert, and J. A. Stamatoyannopoulos. Bedops: high-performance genomic feature operations. Bioinformatics, 28(14):1919-1920, 2012. 
bioRxiv preprint doi: https://doi.org/10.1101/609396; this version posted April 16, 2019. The copyright holder for this preprint (which was not certified by peer review) is the author/funder, who has granted bioRxiv a license to display the preprint in perpetuity. It is made available under aCC-BY-ND 4.0 International license.

\section{Article short title}

A. R. Quinlan and I. M. Hall. Bedtools: a flexible suite of utilities for comparing genomic features. Bioinformatics, 26(6):841-842, 2010.

S. Cass and P. Bulusu. Interactive: The Top Programming Languages 2018. IEEE Spectrum,2018 https://spectrum.ieee.org/static/interactive-the-topprogramming-languages-2018.

P. Moritz, R. Nishihara, S. Wang, A. Tumanov, R. Liaw, E. Liang, W. P., M. I. Jordan and Ion Stoica, Ray: A Distributed Framework for Emerging AI Applications, arXiv, 1712.05889, 2017 